\title{
Wegner on hallucinations, inconsistency, and the illusion of free will. Some critical remarks
}

\author{
Gerben Meynen
}

Published online: 8 June 2010

(C) The Author(s) 2010. This article is published with open access at Springerlink.com

\begin{abstract}
Wegner's argument on the illusory nature of conscious will, as developed in The Illusion of Conscious Will (2002) and other publications, has had major impact. Based on empirical data, he develops a theory of apparent mental causation in order to explain the occurrence of the illusion of conscious will. Part of the evidence for his argument is derived from a specific interpretation of the phenomenon of auditory verbal hallucinations as they may occur in schizophrenia. The aim of this paper is to evaluate the validity of the evidence on auditory verbal hallucinations as employed by Wegner. I conclude that auditory hallucinations do not provide solid evidence for Wegner's theory. Moreover, the phenomena in schizophrenia provide, in fact, an argument against part of Wegner's theory of apparent mental causation.
\end{abstract}

Keywords Wegner Auditory hallucinations · Free will · Incoherence · Psychopathology $\cdot$ Philosophy

\section{Introduction}

Wegner's argument about the illusory nature of conscious will, as developed in The Illusion of Conscious Will (2002) and other publications (Wegner 2003, 2004), has had major impact and it has received much approval. Dennett (2003, p. 224), for instance, says: "I think Wegner's account of conscious will is the best I have seen. I agree with him in almost every regard. (...) He is showing that conscious will is an illusion." In Wegner's project, it is vital that he approaches conscious will as an experience. In fact, the first step in Wegner's project is to understand conscious will as a feeling and the second step is to unmask this feeling as illusory.

In order to show its illusory nature, Wegner not only provides many examples of situations in which we think we do something consciously, while we do not (see also

\section{G. Meynen $(\varpi)$}

Faculty of Philosophy, EMGO Institute VU Medical Center, VU University Amsterdam,

De Boelelaan 1105, 1081 HV Amsterdam, The Netherlands

e-mail: g.meynen@ph.vu.nl 
Mele 2008, p.119). He also develops a theory of 'apparent mental causation' (see, e.g., Wegner 2002, p. 144). This theory tries to explain when and why we do experience our actions as 'freely willed'. ${ }^{1}$ For Wegner wants to be able to identify the very circumstances under which the illusory feeling of free will arises. According to Wegner, "If we begin with 'voluntary behavior can occur without conscious will' and so accept that people construct an experience of will for much of their voluntary action, we must then explain the common co-occurrence of intention and action." (Wegner 2002, p. 143) So, developing a sound theory of apparent mental causation, which explains this common co-occurrence, is a necessary part of his project.

Via the following imaginative situation, Wegner (2002, p. 63) arrives at the three components of his theory of apparent mental causation: "Imagine for a moment that by some magical process you could always know when a particular tree branch would move in the wind. Just before it moved, you would know it was going to move, in which direction, and just how it would do it. Not only would you know this, but let's assume that the same magic would guarantee that you would happen to be thinking about the branch just before each move. You'd look over, and then just as you realized it was going to move, it would do it. In this imaginary situation, you could eventually come to think that you were somehow causing the movement." Next, Wegner (2002, p. 69) argues that in case the branch moved before the thought, or if "you thought of the tree limb's moving and then something quite different moved (say, a nearby chicken dropped to its knees)", or in case you "thought of the tree limb's moving but noticed that something other than your thoughts had moved it (say, a squirrel), no will would be sensed." observations point to three key sources of the experience of conscious will." In order to experience apparent mental causation, there are three requirements (Wegner 2002, p. 69). First, the thought should occur before the action (priority criterion). Second, the thought should be consistent with the action (consistency criterion), and third, it should not be accompanied by other potential causes (exclusivity criterion; Wegner 2002, p. 69). When these criteria are met, then our mind fabricates the (illusory) experience of conscious will as the source of this particular behavior. Wegner (2002, p. 96): "Because we have thoughts of what we will do, we can develop causal theories relating those thoughts to our actions on the basis of priority, consistency, and exclusivity. We come to think of these prior thoughts as intentions, and we develop the sense that the intentions have causal force (...)". According to Schultz et al. (2004), "[t]he strongest version of Wegner's claim would be that priority, consistency, and exclusivity are both necessary and sufficient for the experience of willing an action." It is the second criterion, about consistency, in which Wegner discusses the phenomenon of auditory verbal hallucinations. He uses this phenomenon as evidence ('clinical evidence' Wegner 2004) for the consistency requirement, and thus for his theory of apparent mental causation.

\footnotetext{
${ }^{1}$ In this paper, I will assume Wegner's argument to be not just about 'conscious' will, but (also) about free will. This is in line with Wegner's own interpretation (2002, p. 318) and with interpretations of Wegner's work (see also the Open Peer Commentary to the Précis 2004, and Shariff et al., 2008, and Dennett 2003, as cited above). I have to note that I will not discuss whether a conscious will and a free will should indeed be considered to be identical.

${ }^{2}$ Wegner 2002, p. 69. Also referring to Wegner and Wheatley (1999). My emphasis.
} 
In this paper, I will examine this evidence and I will conclude that auditory verbal hallucinations cannot be considered to deliver the evidence Wegner wants them to provide for his theory. The outline of the paper is as follows. In the next section, I will discuss Wegner's account of instances of creative insight in (brilliant) scientists, sportsmen, and artists, which Wegner understands as phenomena that can be characterized as 'inconsistent'. In "Hearing voices" section, I will present Wegner's view on auditory hallucinations, and more precisely, how he understands these phenomena as related to 'inconsistency' via a theory on auditory hallucinations put forward by Hoffman (1986). Wegner's position will be confronted with, and criticized on the basis of, current literature on auditory hallucinations. "Inconsistency and voices in schizophrenia" section contains my main criticism on Wegner's view of auditory hallucinations and inconsistency, also by relating his view on hallucinations to his earlier account of creative insight. I will argue that, in fact, the phenomenon of inconsistency in schizophrenia does not deliver the evidence for his theory Wegner suggests. It even provides an argument against the consistency requirement of Wegner's theory of apparent mental causation.

\section{Consistency and "Eureka!"}

Wegner (2002, p. 79) explains the term consistency as follows: "When people do what they think they were going to do, there exists consistency between thought and act, and the experience of will is enhanced. ("I thought I'd have a salad for lunch, and here I am eating it.") When they think of one thing and do another-and this inconsistency is observable to them - their action does not feel as willful. ("I thought I'd have a salad for lunch, and here I am with a big plate of fries.")" Consistency of thought and action depends on a 'cognitive process' whereby the thoughts occurring prior to the act are compared to the act as subsequently perceived. (Wegner 2002, p. 79)

Now, according to Wegner (2002, p. 81), "the happiest inconsistency" between intention and action occurs when a great idea pops into mind: "The "action" in this case is the occurrence of the idea, and our tendency to say "Eureka!" or "Aha!" is our usual acknowledgment that this particular insight was not something we were planning in advance." Apparently, while Wegner speaks here about thought/action consistency, he has also thought/thought consistency in mind. ${ }^{3}$ In Wegner's view, while most of us are quite willing to take credit for our good ideas, it is still true that we do not experience these good ideas as 'voluntary'. They happen to us, jumping to our mind: "The writer finds ideas hopping onto the page, the violinist finds musical ideas sneaking into the bow, the tennis player finds inspirations enlivening the racket. In each of these cases, the sense of willfulness of the action is curiously

\footnotetext{
${ }^{3}$ From the last quote, it is clear that Wegner indeed considers 'thought-thought' conditions to fit into his general thought-action scheme (see also what follows in this section and in "Hearing voices" section). However, in literature on hallucinations and thought insertion, it has been emphasized that thought-action and thought-thought relationships should be distinguished, see, e.g., Stephens and Graham (2000) and Gallagher $(2004$, p. 11, 14). See footnote 12 on problems that arise when using a (conscious) thoughtaction scheme in the context of thought-thought relations. Wegner, however, does not explain his position in this debate. In this paper, I will focus my criticism on inconsistency and hallucinations, and not on his use of a thought-action scheme within a thought-thought context.
} 
absent from an act that seemed eminently willed. Although one may have desperately desired to have an insight or a cool move, a fully developed thought of what it would be did not appear in mind in advance, and so there is little sense of authorship when it finally arrives." (Wegner, 2002, p. 82) So, the suggestion is that all of these creative and valuable ideas would be inconsistent with prior thoughtsjust popping up in our mind-and yet we are willing to take credit for them.

Wegner also provides two specific examples of great ideas that are, in his view, neither 'voluntary' nor 'consistent'. He cites the mathematician Henri Poincaré who says:

"I left Caen, where I was then living, to go on a geologic excursion under the auspices of the school mines. The changes of travel made me forget my mathematical work. Having reached Coutances, we entered an omnibus to go some place or other. At the moment when I put my foot on the step the idea came to me, without anything in my former thoughts seeming to have paved the way for it...(...). One morning, walking on the bluff, the idea came to me, with just the same characteristics of brevity, suddenness, an immediate certainty." (Wegner 2002, p. 82, see also Poincaré 1982, p. 387-8)

The second example is derived from Albert Einstein, who is cited by Wegner (2002, p. 83):

"I was sitting in a chair in the Patent Office at Berne when all of sudden a thought occurred to me: 'If a person falls freely he will not feel his own weight.' I was startled. This simple thought made a deep impression on me. It impelled me toward a theory of gravitation."

Wegner concludes from the accounts of these two geniuses that creative insights differ from other ideas primarily in the qualities of suddenness and unexpectedness: "Without a preview in thought, then, the creative leap feels more like a gift than something we have consciously willed." (2002, p. 83) And "[t]hese observations suggest that people are likely to experience little willfulness for some of their most inspired actions - the peaks of their creativity and skill. These actions spring into existence with seemingly minimal conscious thought (...) and so can present to us a sense of being unwilled. It is ironic that many of the most skilled acts we can do, the actions that others enjoy or find simply marvelous because of their high level of skill, (...) may not be things we experience willing."

In my view, it is quite understandable what Wegner is aiming at: a sort of spontaneity or 'inspiration'. Yet, what is apparently vital to Wegner's account is to understand these phenomena as inconsistent thoughts and actions. Basically, we can ask ourselves: are these Eureka moments really instances of inconsistency? "Eureka!" means: "I found it!" Finding does not necessarily follow from searching, but it is also not inconsistent with searching. There is not only no fundamental inconsistency between searching and finding, 'Aha' or "Eureka!", in my view, in fact often points to a profound consistency: the previous thoughts fall in their places. "Eureka!" indicates usually the arrival at something profoundly consistent with

\footnotetext{
${ }^{4}$ Yet, Poincare himself emphasized the vital role of conscious effort in the creative process (see Poincaré 1982, p. 389).
} 
earlier deliberations, and aspirations. It is in fact likely to provide further consistency to earlier thoughts and observations. And although Poincaré's idea may be unexpected, and although Einstein's idea may be unanticipated and unforeseen, it was, not inconsistent with their passionate pursuing, nor inconsistent with their previous work or the conceptual issues they were contemplating. As I understand Wegner, 'consistency' requires a high level of control, calculability, and predictability about what thoughts arise and when they will arise. Consistency, in Wegner's sense, would imply: no real surprises.

In order to further corroborate the consistency requirement, Wegner now turns to another instance of inconsistency, as it may occur in schizophrenia. I will discuss Wegner's account of schizophrenia first, and then, in "Inconsistency and voices in schizophrenia" section, I will relate it to his account of geniuses and unexpected thoughts.

\section{Hearing voices}

Auditory verbal hallucinations or "voices" are reported by $60-80 \%$ of persons with schizophrenia at various times during the course of illness (Hoffman 2008), though lower percentages are also mentioned (Sadock and Sadock 2005). They often produce high levels of distress and functional disability that can be difficult to treat (Hoffman et al. 2007), yet, sometimes, they may also be perceived as 'pleasurable' phenomena (Sanjuan et al. 2004).

According to Wegner, "the intriguing aspect of schizophrenic voices is their origin in thought/action inconsistency" (2002, p. 86) and the consistency principle "offers a way of understanding the experiences of involuntariness reported by people with schizophrenia." (Wegner 2002, p. 84) Wegner states that voices seem to arise when the patient's own inner speech is perceived to be coming from outside the self. And this attributing to someone else seems to occur primarily because the episode is inconsistent with prior thought. (Wegner 2002, p. 86-7) Normally, thoughts follow other thoughts. Although we do not need to know everything in advance, usually we have an idea what will come next. But when inconsistent thought or action appears, we lose our sense that we are the ones in charge. "The voices seem to belong to someone else." In general, as Wegner puts it, "Ideas appear that don't fit the prior plan, and when this happens, perhaps it begins to seem reasonable to attribute the thoughts to a "voice"." $(2002,87)$ Wegner adds: "The prevailing theory of auditory hallucinations is based on precisely this line of reasoning."

Wegner refers to a study on discourse planning disturbances schizophrenia performed by Hoffman that would provide evidence for his theory. Hoffman had developed assessments of "discourse deviations"- departures from a plan for what to say - that may occur in people's answers to interview questions. (2002, p. 87) Wegner (2002, p. 87-88, see also Hoffman 1986) provides an example of such discourse deviations:

Interviewer: Tell me about school. Patient: "School? Well there are schools of play and schools of fish, mostly you see fish school, people edumacating [sic] themselves, you see, sea is one thing and education is another. Fish is school in 
their community, that's why the community of man stands in the way of the community of the sea, and once they see the light of the sunny sunshine then they well let it be."

Wegner comments that this answer seems to involve "puns and plays on words with no overall goal". He adds that Hoffman found that people with schizophrenia who had verbal hallucinations had much higher scores on discourse deviation than those who did not experience verbal hallucinations. "This suggests that the attribution of one's thoughts to "voices" may arise from the failure of those voices to follow a consistent plan that would help the words to seem willful." (Wegner 2002, p. 88)

The overall picture Wegner describes is the following. There is a cognitive process that screens thoughts for their consistency with previous thoughts, and if a thought is insufficiently consistent with previous thoughts, then it is 'interpreted' as stemming from an external source and (consequently) its contents is experienced as an alien voice. As long as thoughts are considered consistent by this system, an experience of free will is fabricated as an add-on. According to this view, free will is an experience secondary to a consistency-driven attribution mechanism. Inconsistent elements (thoughts) lead to the suspension of the fabrication of the add-on experience of these elements being 'freely willed'. "Voices" in schizophrenia are referred to as a phenomenon supportive of this view.

Wegner's account, in fact, builds upon part of Hoffman's 1986 proposed model. ${ }^{5}$ Yet, it is important to note that although Hoffman's proposal has been considered to be intriguing and provoking, it is not the 'prevailing' view on the pathophysiology of auditory verbal hallucinations. It was Hoffman's - interesting-hypothesis.

One of the current views on the pathogenesis of auditory hallucinations concerns (indeed) disorders of inner speech. This inner speech could, due to monitoring problems, it is hypothesized, not be recognized as self/generated, and consequently be heard as voices (see, e.g., Vercammen et al. 2007, Frith 1992, Blakemore et al. 2000 and David 1999). Frith (1992, p. 73) for instance says: "I will now introduce a concept that is very central to my own account of positive symptoms [in schizophrenia]: self-monitoring. If hallucinations are caused by inner speech, then the problem is not that inner speech is occurring, but that patients must be failing to recognise that this activity is self-initiated. The patients misattribute self-generated actions to an external agent." Campbell $(1999,611)$, inspired by Frith and building on Held (1961), supposes that, "when a motor instruction is sent for bodily movement, a copy of that instruction - the "efference copy"-is also sent to some other centre." Via this efference copy, this other center can 'monitor' or check whether that instruction is appropriate and works out well. On Campbell's (1999, p. 611-612) account it could be that our sense of agency grounds in a match at the level of the comparator between the efferent copy and the sensory feedback about the actual movement. In this way, self-monitoring could lead to a sense of agency. It is important to note that the comparator also functions to process motor intentions, so not actual movement, but intended movement. This enables the system to

\footnotetext{
${ }^{5}$ Hoffman's (1986) paper does not speak about 'inconsistency', but about 'incoherence' and 'unintendedness' (see, e.g. 1986, p. 510). Wegner, however, does not explain why he does not use the term 'incoherence' that is central in Hoffman's account, but, instead, speaks about 'inconsistency'.
} 
anticipate future states, at a point in time where no sensory data are present about the outcome of the (intended) movement.

An impairment in a 'self-monitoring' mechanism could also explain and interesting finding by (Blakemore et al. 2000). They observed that patients with auditory hallucinations and/or passivity experiences showed no difference in their perception of self-produced and externally produced tactile stimuli. This was in contrast to patients without auditory hallucinations/passivity experiences and control subjects, who perceived self-produced tactile stimuli as less intense, tickly, and pleasant than externally produced tactile stimuli. Blakemore et al. found that it was the presence of hallucinations and/or passivity experiences, rather than diagnosis (schizophrenia) that was associated with this outcome. They interpret their findings as suggesting that patients who have hallucinations and/or passivity experiences have an abnormal awareness of the sensory consequences of their own movements, which is in line with a self-monitoring hypothesis (see also Frith et al. 2000).

It is important to note that current self-monitoring theories do not involve the idea that voices are heard because of some inconsistency. In fact, Frith (1992, p. 75) already mentions Hoffman's theory on incoherence, yet he argues that "[d]irect empirical support for this [Hoffman's] theory is lacking because, in most studies (...) no associations are found between hallucinations and incoherence of speech. A patient with auditory hallucinations is neither more nor less likely to be incoherent than any other patient." So although Frith's theory and Hoffman's have "much in common" (Frith 1992, p. 105), the difference is that Hoffman's theory relies on incoherence, which, in turn, is also central to Wegner's project on (in)consistency as related to the illusion of conscious will. So, based on current literature, we can conclude that Wegner's presentation of Hoffman's hypothesis as the 'prevailing' theory is not justified. ${ }^{6}$ In fact, the origin of auditory hallucinations is, at present, not clear. In the next section, I will evaluate Wegner's interpretation of incoherence in schizophrenia and hearing voices, and also relate his account to unexpected thoughts or 'inspiration'.

\section{Inconsistency and voices in schizophrenia}

I will develop three lines of criticism. First, I will point to problems that arise when we take other psychopathological phenomena that may occur in schizophrenia into account. Second, I will argue that if we would assume that Wegner is right, then an inconsistency arises in his account of geniuses on the one hand and voices in schizophrenia on the other. Third, I will argue that the phenomenon of inconsistency in schizophrenia provides us in fact with an argument against the consistency requirement of Wegner's theory of apparent mental causation.

I have to note that I will basically discuss Wegner's account-not Hoffman's. Meanwhile, some of the points I bring forward with respect to Wegner in "Wegner's view and other psychopathological phenomena" section were already brought forward against Hoffman (1986).

\footnotetext{
${ }^{6}$ See Allen et al. 2007 for arguments against the monitoring hypothesis. See Jones and Fernyhough (2007) for an attempt to use Wegner's view in an account of hearing voices.
} 
Wegner's view and other psychopathological phenomena

Wegner holds that auditory verbal hallucinations occur when thoughts are considered inconsistent. So, the idea is that we have some consistency meter that considers some of our thoughts inconsistent and then lets them be heard as voices. Yet, in schizophrenia, the phenomenon of 'thought insertion' might occur. This is, according to Sadock and Sadock (2005, p. 1420), a feeling that other people are intruding their thoughts on the patient. Wegner mentions thought insertion (Wegner 2002 , p. 85), but he does not discuss why inconsistency of thought would especially lead to hearing thoughts as voices from external sources, instead of experiencing them, for instance, 'just' as external or inserted. Gallagher (2004) emphasizes that in case a thought is unbidden (as may often occur in our mental lives), it is even not clear why it would be experienced as 'inserted' (see "Geniuses and voices" section). In the same line of thought, we can certainly ask why such unbidden, 'unexpected' thoughts would be experienced as voices.

In addition, people can also hear voices aloud while considering them their own, a phenomenon called "audible thoughts" (Sadock and Sadock 2005, p. 1420). So, hearing things aloud does not necessarily mean that they are perceived as alien (cf. Reed 1986, p. 531). Therefore, one could argue, hearing things aloud is not per se a 'mechanism of the mind' to show that they have external sources.

\section{Geniuses and voices}

But let us now assume that Wegner is right that inconsistency of thoughts leads to auditory verbal hallucinations. And let us relate this back to the "non-pathological" cases (e.g., Einstein and Poincaré). Wegner has tried to show that two geniuses, Poincare and Einstein, were experiencing 'inconsistent' thoughts. Yet, these brilliant brainwaves were not heard as voices. ${ }^{7}$ At least, Poincaré and Einstein did not mention that their scientific breakthroughs came as auditory verbal hallucinations. So, if they are examples of (great) inconsistency and if a mechanism is in place that fabricates the experience of free will in cases of consistency and that makes thoughts being heard as voices in case of inconsistency, why are not the breakthroughs heard as voices?

What Wegner in fact has done in the section about "Aha!" and creative insight is that he has tried to show that inconsistency is a feature of our thinking as humans as such. Then, why do we not hear voices more often? Wegner, in short, does not address what might be different in the non-pathological condition compared to the (pathological) hallucination condition. We could, of course, try to repair or to supplement Wegner's theory with an additional mechanism. For instance, we could argue that there is a three-step (pathological) process in schizophrenia. The first step would be the occurrence of incoherence, the second the experience of lack of authorship, and the third step making the thoughts heard as alien voices (for the

\footnotetext{
${ }^{7}$ Cf. Allen (1986, p. 518) who says that Hoffman's model "takes it for granted that certain unintended cognitions should be presented as auditory images. They are, he asserts, a common occurrence. Yet auditory images are not presented as a common experience in the research concerning the occurrence of unintended cognitions in normals, in obsessions, and in depression and anxiety states (...).”
} 
importance of the third step see Stephens and Graham 2000 and Gallagher, 2004). In principle, such a cascade could be proposed, yet, it is rather far-fetched if it has to be assumed in order to save the consistency requirement in Wegner's theory on the illusory nature of free will.

Here, I would also return to my comments in the section about creative insight: are these creative insights really inconsistent in the way the discourse deviation in schizophrenia can be considered inconsistent? Wegner seems to identify 'unexpectedness' (a normal part of our mental lives) and 'incoherence' (as in discourse deviation). Yet, in my view, a highly consistent thought may come unexpected. ${ }^{8}$ It is useful not to simply identify the incoherence that may occur in schizophrenia with the unexpectedness of some brilliant - or more common - thoughts. ${ }^{9}$ These are different phenomena, and Hoffman's vocabulary of 'incoherence' in some cases of schizophrenia and unexpectedness or even unanticipatedness of brilliant thoughts is preferable.

\section{Inconsistency in schizophrenia}

Andreasen and Black (2006, p. 576) define incoherence as: "Lacking in unity or consistency; often applied to speech or thinking that is not understandable because of any of the following: lack of logical connection between words or phrases; excessive use of incomplete sentences; many irrelevancies or abrupt changes in subject matter; idiosyncratic word usage; or distorted grammar." This phenomenon could indeed be considered a form or rather an extreme form of 'inconsistency'. Incoherence occurs in more than $20 \%$ of the schizophrenia patients, but it could also occur in other disorders, like during manic episodes in bipolar disorder (Andreasen and Black 2005). Incoherence of speech or thought can be observed by others, but, as for instance Barrera et al. (2008) show, also by schizophrenia patients themselves. As far as we know, incoherence (or formal thought disorder) in schizophrenia is, in principle, present without the feeling that these thoughts do not belong to oneself or that they are alien etc. Incoherence is 'just' incoherence, as defined by Andreasen and Black (2005), although it may be defined and experienced in many ways (Barrera et al. 2008, Elvevåg et al. 2007). This phenomenon, therefore, shows first of all that inconsistency as such does not necessarily lead to a suspension of the sense of 'ownership' or 'authorship' like Wegner's theory of apparent mental causation would predict. ${ }^{10}$ As O'Brien and Opie (2003, p. 119) point out, people suffering from schizophrenia, "and others who suffer quite radical breakdowns in the connectedness and continuity of their experience generally have no doubt that these

\footnotetext{
${ }^{8}$ Verbal hallucinations as it seems cannot be considered merely inconsistent, for they do seem to have their own coherence. They follow semantic rules, and follow a line of reasoning. See Hoffman et al. (1999) and Allen (1986, p. 518) and Rund (1986, p. 531), who emphasizes that voices do not speak in an incoherent manner.

${ }^{9}$ Meanwhile, although there are conflicting results, some studies found a relationship between schizophrenia and creativity (see Abraham et al. 2007 for an overview) and between schizotypy and creativity (Folley and Park, 2005). So, in principle, creative insight (of geniuses and artists) and schizophrenia/schizotypy could somehow be related.

${ }^{10}$ Still, there are also many patients who experience that they are not the author of (some) of the thoughts they experience. Why this is the case remains an area of empirical and phenomenological research (see, e.g., Blakemore et al. 2000, Stephens and Graham 2000, Gallagher 2004).
} 
anomalous experiences belong to them. The representational incoherence of the parts of experience doesn't appear to undermine the phenomenal 'togetherness' that characterizes a single subject of experience."11 In fact, passivity phenomena, which are often understood in terms of changed experience of 'agency' are distinct phenomena from formal thought disorders (see Leube et al. 2008). So, we know that in schizophrenia, there is incoherence but this is a phenomenon that is as such distinct from hallucinations, and from passivity phenomena. Consistency, as far as we know, does not appear to be a requirement for thoughts to be experienced as 'belonging to oneself' or 'self-generated'.

In addition, Jones and Fernyhough (2007, p. 397) say that "there is no evidence that it is these out-of-the-blue thoughts that those diagnosed with psychosis experience as auditory verbal hallucinations." So, there is no evidence that the outof-the-blue thoughts are in fact the semantic entities which are heard as voices because of their out-of-the-blue or inconsistent character. Empirical evidence, of course, is exactly what Wegner is looking for (see also Wegner 2004) in his theory of the illusion of conscious will. And at this point it is lacking. I am not saying that further research could not reveal a relationship that we currently do not know of, but current theorizing should, in my view, be based on what we know now.

To sum up, I emphasize another feature of schizophrenia than Wegner does. In his account of the consistency requirement, Wegner explores a hypothesis on the nature of auditory hallucinations as formulated by Hoffman in 1986. I do not focus on his hypothesis about auditory hallucinations, but on the phenomenon of incoherence in schizophrenia directly. And this leads to an opposite view compared to Wegner. While Wegner holds that psychopathology in schizophrenia provides evidence for the consistency requirement of his theory of apparent mental causation, I conclude that it more directly provides an argument against it.

Another issue concerns the question whether the processes described by Wegner would take place at a conscious of unconscious level. Wegner does not explicitly address this point. Should the process be meant as unconscious, then Wegner could be vulnerable to Gallagher's (2004) criticism of Frith's (1992) theory. Gallagher (2004) argued that it seemed "odd" to assign such a "task to a subpersonal, nonsemantic mechanism when, simply put, we are already consciously aware of our thoughts and can keep track of them, and keep them on track, at a conscious level." In other words, it seems strange that a subconscious process should monitor semantic coherence which is also accessible to consciousness. ${ }^{12}$ Yet, since Wegner

\footnotetext{
${ }^{11}$ There are also different subcategories in the DSM-IV for paranoid schizophrenia (often accompanied by voices) and disorganized schizophrenia (often accompanied by incoherent speech or thoughts; see also Elvevåg et al., 2007).

${ }^{12}$ In fact, according to Gallagher, if the comparator/monitoring/metarepresentation process would indeed involve "a fullfledged act" of conscious reflection then threatens infinite regress. Gallagher: "Since metarepresentation is itself a thought, if I want to attribute this metarepresentation to myself, I would need to monitor it with a meta-metarepresentation. I would have to ask, in a further metarepresentational thought: is this my metarepresentation? An extra level of consciousness would be added to the comparator's verification process. My intention to metarepresent would have to generate its own efference copy, to be matched up on top of the original match." On Gallagher's account, "in the case of thinking, which is already prereflectively conscious, even one level of metarepresentation for maintaining a sense of agency, or even one level of efference copy for purposes of keeping track of thoughts is redundant." On efference copy, see Campbell 1999, also discussed in "Hearing voices" section.
} 
himself does not address this point of the conscious or unconscious nature of the consistency meter keeping track of thoughts, I will not go into it.

\section{Examining Wegner's evidence}

In this paper, I have examined a piece of evidence Wegner provides for his theory of apparent mental causation (as mentioned in Wegner and Wheatley 1999, Wegner 2002, 2003, 2004). Wegner, however, intends to provide much empirical evidence for his claims, which he himself considers crucial to his project. For instance, in the Précis of The Illusion of Conscious Will in Behavioral and Brain Sciences he says: "This précis of The Illusion of Conscious Will is an abridgement of three of the book's chapters. It focuses on the main arguments, and leaves aside the bulk of the empirical evidence relevant to these arguments. The evidence is essential and extensive, however, and the arguments cannot be evaluated effectively without it. Like a vertebrate stripped of its skeleton, this article does not stand on its own."

In order to assess Wegner's influential argument, one would, then, have to look at the "bulk of empirical evidence". And, indeed, Wegner provides many empirical data, not just derived from (his own) psychological research, but also from phenomena like table turning, automatic writing, hypnosis, and remarkable cases reported by neurosurgeons. It is hard to assess in one paper the scientific solidity and validity of all the empirical data Wegner provides and to weigh it against my objections to the consistency requirement. In this paper I have zoomed in on the empirical or 'clinical' evidence as provided by Wegner concerning the phenomenon of auditory verbal hallucinations.

We have seen, first, that auditory hallucinations do not provide the clinical evidence Wegner wants them to provide. For Wegner's account turns out not to be an interpretation of the 'prevailing' theory on this phenomenon. Considering Wegner's account of the origin of hallucinations, many objections could be formulated. In itself, however, this could not be too much of a problem for Wegner's theory of apparent mental causation, at least as long as there is enough other evidence in place to solidly support the consistency requirement. So, in principle, my first line of criticism could be rebuked by providing evidence from other phenomena (other than hallucinations and incoherence in schizophrenia). For instance, it might be that evidence derived from Wegner's own psychological experiments can provide the evidence, after all.

Second, I showed that if Wegner's account would be right, there would be an unexplained discrepancy between inconsistency in schizophrenia and in cases of brilliant insight. This is a problem within his theory that cannot simply be circumvented by providing evidence from other phenomena like hypnosis. Wegner has to address the question why (highly) 'inconsistent' thoughts in mathematicians (Poincaré), physicists (Einstein), and authors are not perceived as alien 'voices', while in schizophrenia inconsistency would be the cause that semantic entities are heard as voices. I added that in my view, creative insight and incoherence should not be considered as both 'inconsistent' in the first place. Brilliant thoughts and incoherence of thought are different phenomena.

Third, for all we know, the phenomenon of incoherence in schizophrenia does not in itself lead to suspension of the experience of authorship or ownership. As far as 
Wegner intends to present incoherence (discourse deviation) in schizophrenia as an extreme form of inconsistency, we could argue that even in such an 'extreme' case of inconsistency, there is no suspension in the sense that semantic entities are heard as voices. This provides us with an argument against the claim that 'consistency' as such is required for some sort of 'authorship experience', or that inconsistency leads to suspension of such an experience.

In fact, much on auditory hallucinations and their origin is not known. And because much is not clear, one has ample opportunity to try to defend Wegner's claims by referring to speculations about how it could be. But I think that such speculations are not the type of entities Wegner wants to build his theory on. Neither are they the kind of entities Wegner is considered to rely on (see also Pockett et al. 2006). Therefore, the lines of criticism as developed above are relevant to Wegner's project.

\section{Conclusion}

Wegner's endeavor linking a psychopathological phenomenon to the 'philosophical' issue of free will is gripping. Yet, auditory hallucinations do not provide solid evidence for Wegner's theory of apparent mental causation, and in this sense, they do not support Wegner's overall claim that free will is an illusion. Moreover, the phenomenon of incoherence in schizophrenia provides us with an argument against the consistency requirement of Wegner's theory of apparent mental causation. Does this mean that free will is not illusory after all? This conclusion would be too sweeping merely on the basis of our considerations so far. However, within Wegner's project the theory of apparent mental causation is central, and within this theory, consistency is one of the three components. Based on the critical considerations put forward in this paper with respect to psychopathology, it is wise to (re)consider whether we should indeed try to understand our everyday experience that we have 'free will' using Wegner's consistency principle. Consistency in the sense presented by Wegner might not be such a central factor in the phenomenology of free will.

Open Access This article is distributed under the terms of the Creative Commons Attribution Noncommercial License which permits any noncommercial use, distribution, and reproduction in any medium, provided the original author(s) and source are credited.

\section{References}

Abraham, A., Windmann, S., McKenna, P., \& Güntürkün, O. (2007). Creative thinking in schizophrenia: the role of executive dysfunction and symptom severity. Cognitive Neuropsychiatry, 12(3), 235-58.

Allen, H. (1986). A three-component analysis of Hoffman's model of verbal hallucinations. The Behavioral and Brain Sciences, 9, 518.

Andreasen, N. C., \& Black, D. W. (2006). Introductory textbook of psychiatry. Washington DC: American Psychiatric Publishing.

Barrera, A., McKenna, P. J., \& Berrios, G. (2008). Two new scales of formal thought disorder in schizophrenia. Psychiatry Research, 157, 225-234. 
Blakemore, S. J., Smith, J., Steel, R., Johnstone, C. E., \& Frith, C. D. (2000). The perception of selfproduced sensory stimuli in patients with auditory hallucinations and passivity experiences: evidence for a breakdown in self-monitoring. Psychological Medicine, 30(5), 1131-9.

Campbell, J. (1999). Schizophrenia, the space of reasons, and thinking as a motor process. The Monist, 82 (4), 609-625.

David, A. S. (1999). Auditory hallucinations: phenomenology, neuropsychology and neuroimaging update. Acta Psychiatrica Scandinavica, Suppl. 395, 95-104.

Dennett, D. (2003). Freedom evolves. London: Penguin Books.

Elvevåg, B., Foltz, P. W., Weinberger, D. R., \& Goldberg, T. E. (2007). Quantifying incoherence in speech: an automated methodology and novel application to schizophrenia. Schizophrenia Research, 93, 304-316.

Folley, B. S., \& Park, S. (2005). Verbal creativity and schizotypal personality in relation to prefrontal hemispheric laterality: a behavioral and near-infrared optical imaging study. Schizophrenia Research, $80(2-3), 271-82$.

Frith, C. D. (1992). The cognitive neuropsychology of schizophrenia. Hillsdale: Erlbaum.

Frith, C.D., Blakemore, S.J., Wolpert, D.M. (2000). Abnormalities in the awareness and control of action. Philosophical transactions of the Royal Society of London, 355(1404), 1771-1788.

Gallagher, S. (2004). Neurocognitive models of schizophrenia: a neurophenomenological critique. Psychopathology, 37, 8-19.

Held, R. (1961). Exposure-history as a factor in maintaining stability of perception and coordination. The Journal of Nervous and Mental Disease, 132, 26-32.

Hoffman, R. E. (1986). Verbal hallucinations and language production processes in schizophrenia. The Behavioral and Brain Sciences, 9, 503-548.

Hoffman, R. E. (2008). Auditory/verbal hallucinations, speech perception neurocircuitry, and the social deafferentation hypothesis. Clinical EEG and Neuroscience, 39(2), 87-90.

Hoffman, R. E., Rapaport, J., Mazure, C. M., \& Quinlan, D. M. (1999). Selective speech perception alterations in schizophrenic patients reporting hallucinated "Voices". The American Journal of Psychiatry, 156, 393-399.

Hoffman, R. E., Hampson, M., Wu, K., Anderson, A. W., Gore, J. C., \& Buchanan, R. J. (2007). Probing the pathophysiology of auditory/verbal hallucinations by combining functional magnetic resonance imaging and transcranial magnetic stimulation. Cerebral Cortex, 17, 2733-2743.

Jones, S. R., \& Fernyhough, C. (2007). Thought as action: Inner speech, self-monitoring, and auditory verbal hallucinations. Consciousness and Cognition, 16, 391-399.

Leube, D., Whitney, C., \& Kircher, T. (2008). The neural correlates of ego-disturbances (passivity phenomena) and formal thought disorder in schizophrenia. European Archives of Psychiatry and Clinical Neuroscience, 258(Suppl 5), 22-27.

Mele, A. R. (2008). Recent work on free will and science. American Philosophical Quarterly, 45, 107-129.

O'Brien, G., \& Opie, J. (2003). The multiplicity of consciousness and the emergence of the self. In A. S. David \& T. Kircher (Eds.), The Self in Neuroscience and Psychiatry. Cambridge: Cambridge University Press.

Pockett, S., Banks, W. P., \& Gallagher, S. (Eds.). (2006). Does consciousness cause behavior? Cambridge: MIT Press.

Poincaré, H. (1982). The foundations of science. Washington: University Press of America.

Reed, G. F. (1986). When is an image hallucinatory? The Behavioral and Brain Sciences, 9, 530-531.

Rund, B. R. (1986). Verbal hallucinations and information processing. The Behavioral and Brain Sciences, 9, 531-532.

Sadock, B. J., \& Sadock, V. A. (2005). Kaplan \& Sadock's comprehensive textbook of psychiatry (8th ed.). Philadelphia: Lippincott Williams \& Wilkins.

Sanjuan, J., Gonzalez, J. C., Aguilar, E. J., Leal, C., \& van Os, J. (2004). Pleasurable auditory hallucinations. Acta Psychiatrica Scandinavica, 110(4), 273-278.

Schultz, J., Sebanz, N., \& Frith, C. (2004). Conscious will in the absence of ghosts, hypnotists, and other people. The Behavioral and Brain Sciences, 27(5), 674-675.

Shariff, A. F., Schooler, J., \& Vohs, K. D. (2008). The hazards of claiming to have solved the hard problem of free will. In J. Baer, J. C. Kaufman, \& R. F. Baumeister (Eds.), Are we free? Psychology and free will. New York: Oxford University Press.

Stephens, G. L., \& Graham, G. (2000). When self-consciousness breaks: alien voices and inserted thoughts. Cambridge: MIT. 
Vercammen, A., de Haan E. H. F., \& Aleman, A. (2007). Hearing a voice in the noise: auditory hallucinations and speech perception. Psychological Medicine, 1-8.

Wegner, D. M. (2002). The illusion of conscious will. Cambridge: MIT Press.

Wegner, D. M. (2003). The mind's best trick: how we experience conscious will. Trends in Cognitive Sciences, 7, 65-69.

Wegner, D. M. (2004). Précis of the illusion of conscious will. The Behavioral and Brain Sciences, 27(5), 649-692. commentary included.

Wegner, D. M., \& Wheatley, T. (1999). Apparent mental causation: sources of the experience of will. The American Psychologist, 54, 480-492. 